\title{
Research on Communication FM Signal Blind Separation under Jamming Environments
}

\author{
Xu Changyue, Huang Gaoming and Hou Xiaoyang \\ College of Electronic Engineering, Naval Univ. of Engineering, Wuhan, 430000, China \\ 444716022@qq.com, hgaom@163.com, 997145178@qq.com
}

\begin{abstract}
Keywords: Blind signal separation; Jamming source; FastICA algorithm; Communication signal Abstract: Aimed at the influence of all kinds of artificial jamming on communication signals in complex electromagnetic conditions, the technology of communication signal separation based on FastICA algorithm is proposed. The technology can separate the communication signal of interest from the receiver mixed a variety of jamming sources according to the statistical characteristics of the source signal, and guarantee the expedite communication. In the end, the test which based on the frequency modulation signal of message and voice in frequency modulation jamming, amplitude modulation jamming and pulse jamming is simulated, and the result shows it is very good to separate and demodulate the communication signals of message and voice. Besides, we analyze the performance of information restoration based on the information correlation under different signal to noise ratio (SNR) and jamming to signal ratio (JSR).
\end{abstract}

\section{Introduction}

With the increasing complexity of electromagnetic environment, the ability of traditional communication and electronic protection has been unable to adapt to the new environment. The various styles jamming take a great threat to our information system, especially, the electromagnetic jamming from malicious people. In order to eliminate the influence of electromagnetic jamming, the traditional communication system usually make use of several conventional "anti-jamming" technologies, such as making use of extended spectrum to reduce the jamming gain, making use of increased jump speed of hopping frequency to reduce the jamming to improve system performance, and making the use of networking to promote the reliability of communication [1]. However, with the development of jamming technology, these methods all have the limitations of practical application, and the protection effect becomes more and more unsatisfactory.

Blind source separation (BSS) technology which is first proposed in the 1980s, has been widely used in mobile communication, biomedicine and other civil fields, and has been highly regarded in the military field. In the field of anti-jamming communication, Yang Xiao-niu applied the blind source separation to the radio, and successfully separated the signal source from the mixed signal in 2008(single-tone modulation signal and single-tone jamming signal) [2]. In 2009, Liu

Qing-ao applied the FastICA algorithm to the separation of the co-channel signal, and the simulation confirmed that FastICA algorithm could separate the co-channel signal and eliminate the co-channel jamming [3]. In 2014, Chen Li, a graduate student of University of Electronic Science and Technology of China, studied the anti-jamming technology of the blind signal separation in the hopping frequency system[4].

In this paper, we study the way of FM signals separated from three kinds of jamming patterns, such as frequency modulation jamming, amplitude modulation jamming and pulse jamming. By this 
method, it gets the original modulation signal just according to the mixed signals received by equipments without learning samples, and then restores the information you need to transmission after demodulation. Finally, based on the correlation of information, the restoration performance of algorithm under different SNR and JSR is analyzed.

\section{The jamming and signal mixing model}

In Fig. 1, we proposed a model based on the blind signal separation based on FastICA communication system: they launch information on the fixed channel, but malicious parties transmit multiple jamming sources on the same channel at the same time. The blind extractor based on FastICA algorithm using statistical independence to obtain the modulation signal of its own communication signals, and finally gets the communication information through demodulation. Because it takes advantage of a strong and practical nature of communication signals--statistical independence, FastICA algorithm can ignore modulation patterns of various types jamming sources.

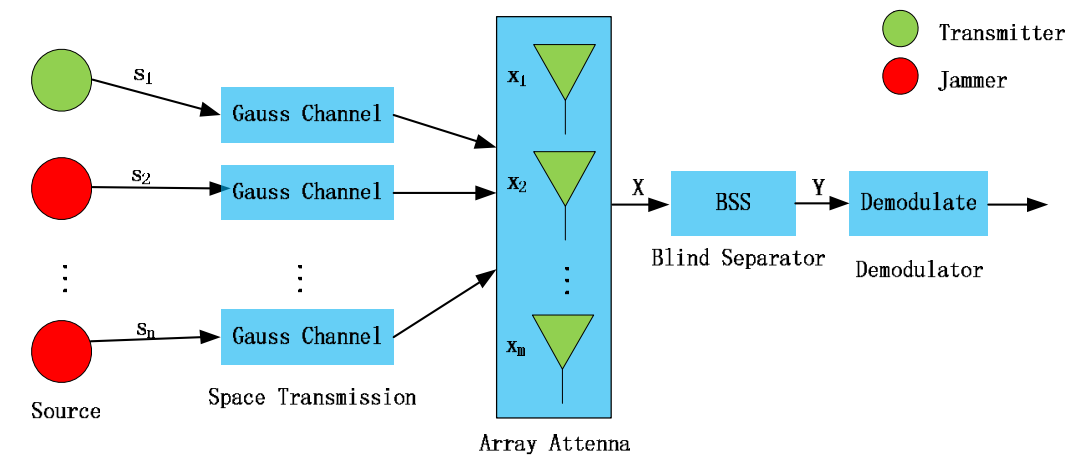

Fig. 1 Model of communication system based on blind separation

The jamming and signal mixing model can be represented as follows[5] :

$$
X=A(S+J)+N
$$

Where $X=\left[x_{1}, x_{2}, \ldots, x_{m}\right]^{T}, J=\left[j_{1}, j_{2}, \ldots, j_{n-1}\right]^{T}, N=\left[n_{1}, n_{2}, \ldots, n_{m}\right]^{T}$ respectively represent $m \times L$ dimension matrix of the observation signal, the $(n-1) \times L$ dimension matrix of the jamming source signals and the $m \times L$ dimension matrix of the additive Gaussian noises; $\mathrm{S}$ represents a $1 \times L$ dimension communication signal; $\mathrm{A}$ is the $m \times n$ dimension stochastic mixed matrix; $\mathrm{L}$ is the number of samples; the upper standard $\mathrm{T}$ represents the transpose; The noise, the communication signal and the jamming source signal are independent where the variance is $\sigma^{2}$.

Output after blind extractor is:

$$
Y=W X
$$

Where $W$ is a demixing matrix, the purpose of the method is to find a $W$ that can make each component of $Y$ as independent as possible, so as to isolate the communication modulated signals of interest $y$, which is the recovered signal of the communication signal $s_{1}$.

\section{FastICA algorithm}

The rapid fixed point ICA (FastICA) algorithm is based on the minimization of negative entropy by using fixed-point to iterate. The algorithm, which is a fast algorithm of independent component analysis, uses the Newton iterative algorithm for batch processing vast sampling points 
of variable $\mathrm{X}$ from observation, and each time it separates an independent component each from signals observed. In order to reduce the estimated parameters and simplify the computation of the algorithm, before running the FastICA algorithm, we need to preprocess the data required, namely, the process of removing mean and whitening. The observed signal is pre-bleached so as to form the orthogonal transformation relationship between the observed signal and the source signal, and then the signal is separated by orthogonal matrix. Therefore, we need to keep the orthogonal properties of the separation matrix the When the negative entropy is minimum. The advantage of the fixed point algorithm is that there is no selection problem of the learning factor, and the convergence speed is very fast, which can realize the single signal separation, or extract several source signals simultaneously.

The FastICA algorithm in noise condition estimating that the hybrid matrix has the following iterative formula [6-7] :

$$
w_{k+1}=E\left\{\ell g\left(w_{k}^{T} \mathscr{l}(d)\right)\right\}_{k}-\left(1+\stackrel{g}{g} g E\left\{g^{\prime}\left(w_{k}^{T} x(t)\right)\right\} w_{k}\right.
$$

Where, $w_{k+1}$ is the new value of $w_{k}$. After each iteration, we need make $w_{k+1}$ normalized to the unit norm. $2^{Q / c}$ is given by the follo wing formula:

$$
\sum^{2}=E\{M \% \%\}=(C-\Sigma)^{-1 / 2} \sum(C-\Sigma)^{-1 / 2}
$$

Besides, in the formula (3), $g$ is the derivative of function $G$, which can be selected from the following functions in the table [8]:

Table 1
\begin{tabular}{|c|c|c|}
\hline NO. & $G(9$ & $g\left(9=G^{\prime}(9\right.$ \\
\hline 1 & $G_{1}(u)=\log \cosh (u)$ & $g_{1}(u)=\tanh (u)$ \\
\hline 2 & $G_{2}(u)=\exp \left(-u^{2} / 2\right)$ & $g_{2}(u)=u \exp \left(-u^{2} / 2\right)$ \\
\hline 3 & $G_{3}(u)=u^{4} / 4$ & $g_{3}(u)=u^{3}$ \\
\hline
\end{tabular}

The specific steps of the algorithm are shown below:

(1) Selected the initial weight vector $w_{0}$, randomly, and let $k=0$;

(2)Update weight vector $w_{k+1}$ using the formula [3].

(3)Normalize $w_{k+1}=w_{k+1} /\left\|w_{k+1}\right\|$;

(4)If $\left|w_{k+1}-w_{k}\right|>\varepsilon$, namely, the algorithm does not converge, return step (2); Otherwise, estimates an independent component, and then end the algorithm.

\section{Simulation and analysis}

\section{The performance of restored signal}

In digital communication, We usually judge the quality of communication transmission by bit error rate, but generally not in analog communication, because the shape of its restored signal and its emission signals' may be the same while with the different amplitudes, so we pay more attention to the shape information. Considering these reasons, in order to quantify the restored performance 
of frequency modulation signal based on blind separation, we use mutual-correlation to describe the degree of restored information.

If the original message is $s(t)$ and the recovered communication signal is $z(t)$, in the time ( $\mathrm{t} 1$, t2), the degree of recovery is:

$$
R=\int_{t 1}^{t 2} s(t) z(t) d t
$$

Where, mutual-correlation $\mathrm{R}$ represents the quality of its signal recovery, ranging from 0 to 1 . In general, the closer the $\mathrm{R}$ is to 1 , the higher the quality of the recovery. On the contrary, the closer the $\mathrm{R}$ is to 0 , the poorer the quality of recovery.

In this simulation, we define the number of independent source signals $n=4$, and assume that the number of signal source is consistent with the observed antenna array signal number, that is $m=n$. The matrix A which is a $n \times n$ dimension random mixing matrix is randomly generated, and set sample size $\mathrm{L}=50000$. This paper uses nonlinear function $g_{3}(u)=u^{3}$. Next, we use the FastICA algorithm to separate mixed data.

\section{The frequency modulation signal of message}

The message signal is a random integer evenly distributed between [-3, 3], and the time interval is $1 / 2 \mathrm{~s}$. The message signal adopts the FM modulated carrier $\cos 2 \pi f_{c} t$, assuming carrier frequency $f_{c}=300$, frequency modulation sensitivity $k_{f}=50$, and the simulation time $0 \leq t \leq 5$. In the same frequency, there are three kinds of jamming patterns, such as frequency modulation jamming, amplitude modulation jamming and pulse jamming. In Fig.2, while signal-to-noise ratio is $30 \mathrm{~dB}$, (a) shows the signal for the required transmission, as well as the corresponding jamming message, and (c) shows the corresponding modulation pattern. The experimental process is shown in Fig. 2 (a-e).

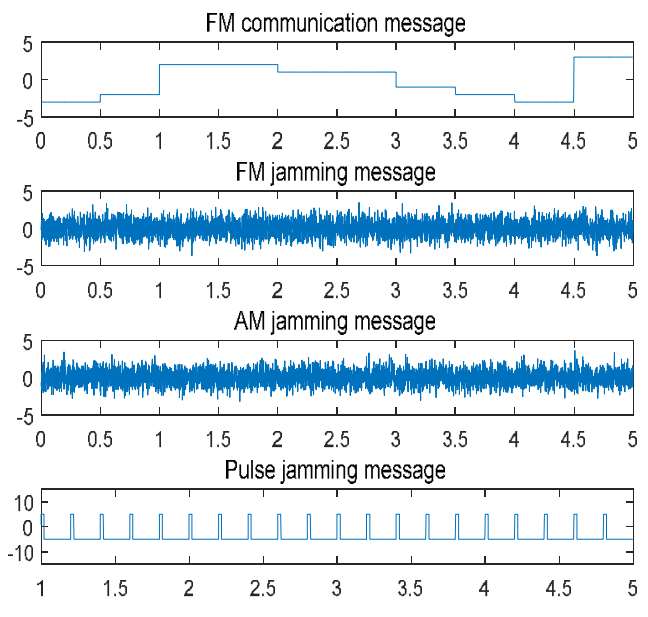

(a) The original messages

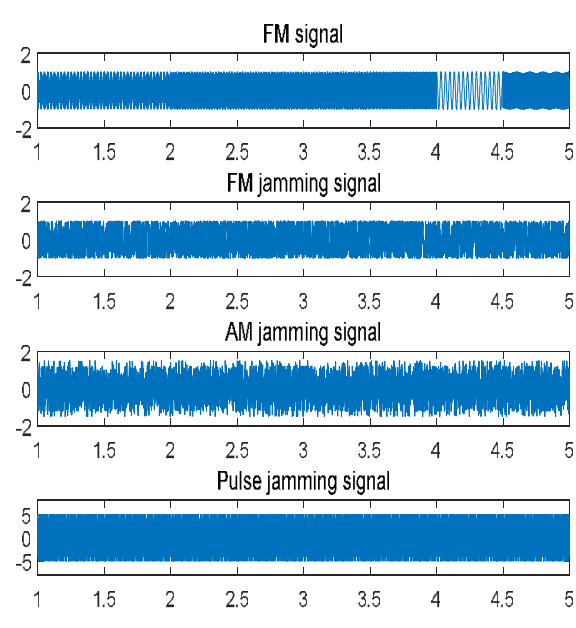

(b) Signals after modulation 


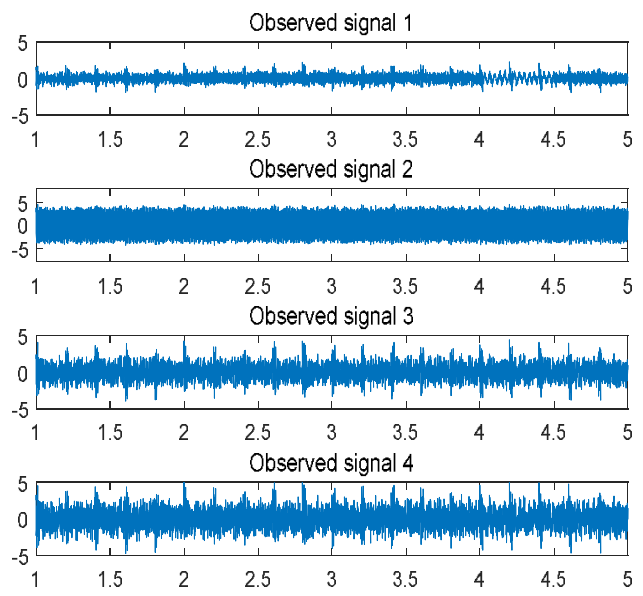

(c) Observed signals from antenna arrays
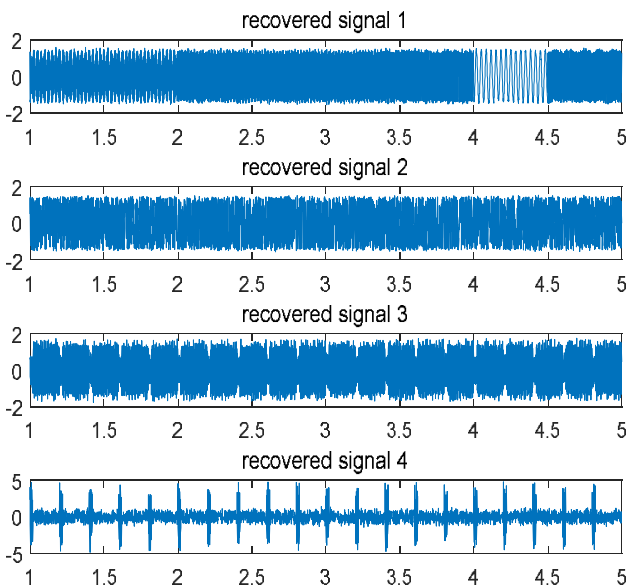

(d) Signals after separated and recovered

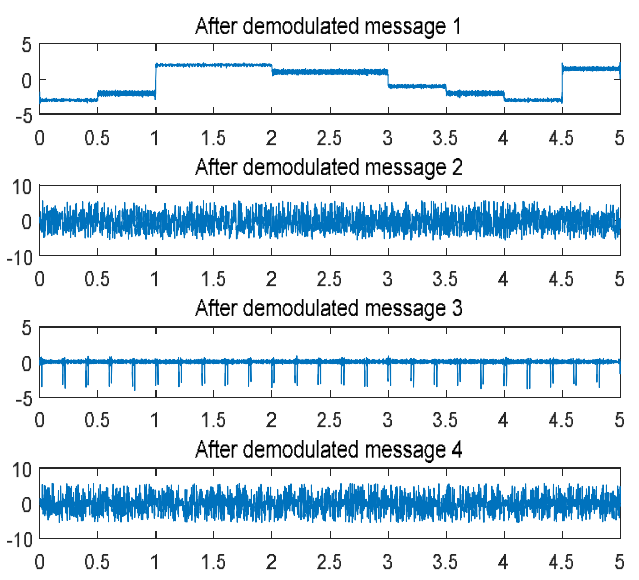

(e) Messages after demodulation

Fig. 2 The simulation process of message frequency modulation signal

The performance of message FM signal algorithm is shown in table 2.We can see from table 2 and Fig. 2, though it is not very ideal to recover other jamming message, but interested communication message is recovered very nice, up to the purpose of the experiment. In addition, the weight vector can be convergent at most about 10 iterations, which the real time is good.

Table 2 The algorithm performance of message frequency modulation signal

\begin{tabular}{|c|c|c|c|c|c|}
\hline SNR & 20 & 30 & 40 & 45 & 50 \\
\hline Iterations & 10 & 9 & 8 & 7 & 7 \\
\hline
\end{tabular}

The restoration performance of the message FM signal varying with different SNR and JSR, is shown in Fig.3, and we use the above correlation to reflect the performance of the recovery. As can be seen from (a), with the increase of SNR, the recovery degree of message is better; when the SNR is below $25 \mathrm{~dB}$, the recovery performance decreases sharply, and unreliability increases; when the signal-to-noise ratio was higher than $25 \mathrm{~dB}$, the recovery performance will be close to the extreme and good reliability. As we can see from (b), with the change of the JSR, it has little influence for message with the small amount of information. In addition, it can be seen that the noise affects the performance of the algorithm more than the malicious jamming, because the superimposed noise affects the independent statistics of the communication signal more easily. 


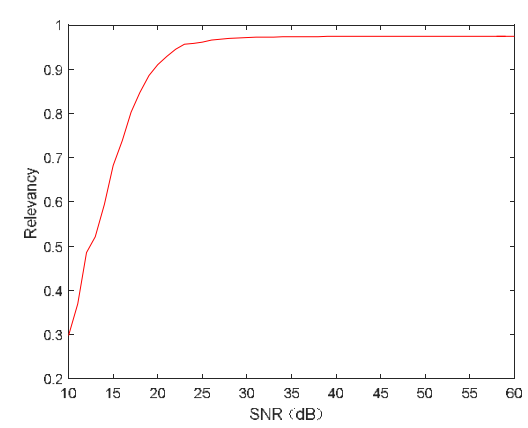

(a) The separation performance of message with different signal-to-noise ratio

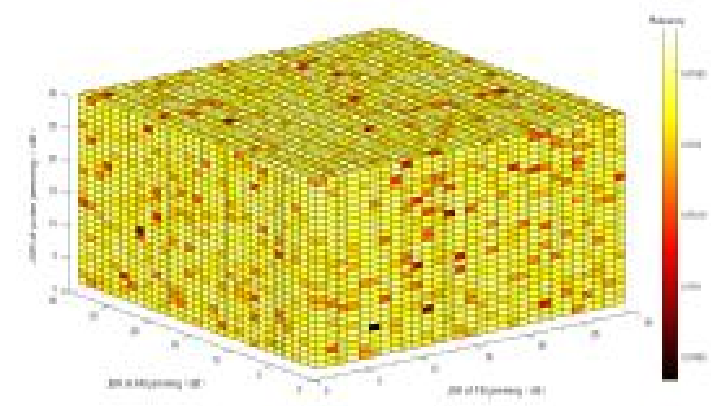

(b) The separation performance of message with different jamming-to-signal ratio

Fig. 3 The separation performance of message

\section{The frequency modulation signal of voice}

Under the above conditions, the separation effect of voice FM signal under these three kinds of jamming is given below, and the process is similar to that of the above message FM signal. While signal-to-noise ratio is $30 \mathrm{~dB}$, Fig. 4 (a-b) respectively gives the original waveform of the voice message in the time domain and frequency domain, as well as the eventual waveform abstracted after jammed. From the Figure, we can be see that the voice message can be restored in the time domain and frequency domain very well. In the actual situation, the voice message that is not directly separated by the blind extractor, is completely submerged in the noise, but the voice can be heard very clearly after the blind extractor and demodulator.

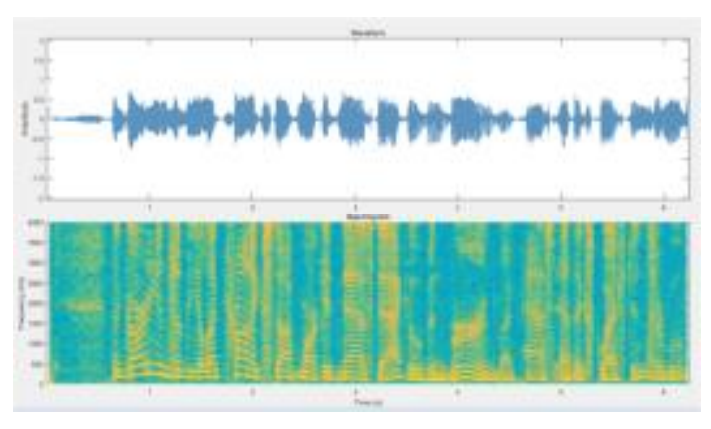

(a) The original voice messages

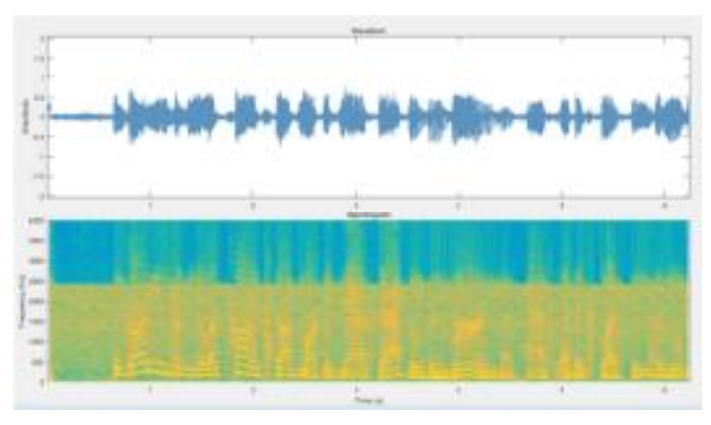

(b) The voice messages after separated and demodulated

Fig. 4 The simulation process of voice frequency modulation signal

Similarly, Table 3 shows the algorithm performance of voice frequency modulation signal, it can be seen that the weight vector iteration times is larger than a message signal's in the table, but it can meet the requirements up to the 15 iterations and the real time changes little.

Table 3 The algorithm performance of voice frequency modulation signal

\begin{tabular}{|c|c|c|c|c|c|}
\hline SNR & 30 & 35 & 40 & 45 & 50 \\
\hline Iterations & 15 & 13 & 12 & 11 & 11 \\
\hline
\end{tabular}

The restoration performance of the voice FM signal varying with different SNR and JSR, is shown in Fig.5, and similarly, we use the above correlation to reflect the performance of the recovery. 


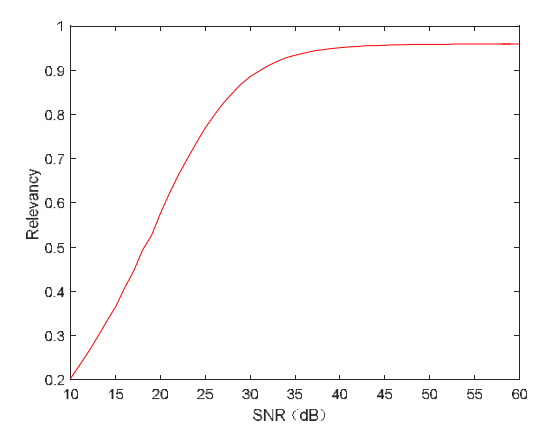

(a) The separation performance of voice with different signal-to-noise ratio

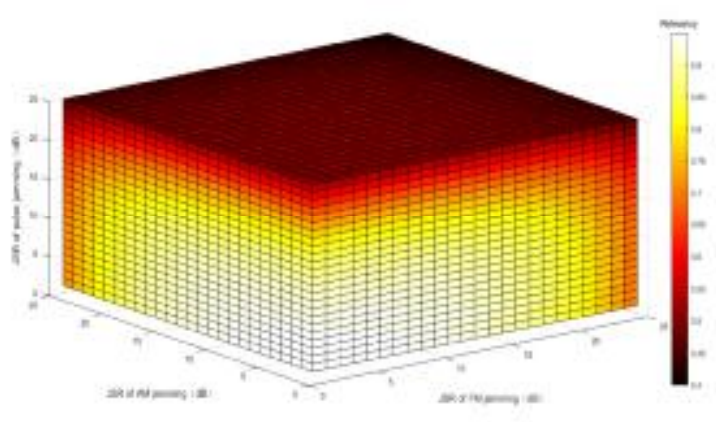

(b) The separation performance of voice with different jamming-to-signal ratio

Fig. 5 The separation performance of voice

As can be seen from (a), with the increase of SNR, the recovery degree of voice is better; when the SNR is below $30 \mathrm{~dB}$, the recovery performance decreases sharply, and the recovery voice is more unclear; when the SNR is higher than $30 \mathrm{~dB}$, the recovery performance close to the extreme, the recovery voice become clear. As can be seen from (b), as the JSR increases, the effect of speech recovery began to deteriorate; from the three dimensions of the (b) axis, the performance is basically the same with the increase of the interference intensity of the three disturbances, which shows that it has little to do with the pattern of interference signals by this method; when the three kinds of jamming SNR are all less than $20 \mathrm{~dB}$, the speech recovery effect is good, but in more than $20 \mathrm{~dB}$, the recovery degree began to decrease. Different from the message signal, the voice with large amount of information is greatly affected by malicious jamming, and the impact of noise is also relatively larger than jamming.

\section{Conclusions}

In this paper, we mainly study the frequency modulation signal under three kinds of jamming sources, and use the FastICA algorithm to extract and demodulate the modulation signal. By the method, it obtains the corresponding observation mixed signals through the array antennas at first, and then recovers the modulation signal based on the FastICA algorithm blind separation, and demodulates the recovery signal at last. Finally, this paper shows the simulation of FastICA algorithm based on blind source separation, which the message and voice information which is required to pass is good restored. Besides, through quantitative analysis using the mutual-correlation characteristics of information in different SNR and JSR, we get the conclusion that the impact of noise is also relatively larger than jamming. The method of communication signal separation based on blind separation provides a new solution to improve the reliability of communication system in complex electromagnetic environment.

\section{Acknowledgements}

This study is supported by the National Natural Science Foundation of China (Grant Nos. 61601491).

\section{References}

[1]Yao Fu-Qiang. Communication Anti-jamming Engineering and Practice[M]. Beijin: Publishing House of Electronics Industry, 2012. 
[2]YANG Xiao-niu, FU Wei-hong. Blind Source Separation:Theory, Application and Prospect[J].Communication Countermeasures, 2006, (3):3-10.

[3]Liu qing-ao. Co-channel Jamming Restraint Using Blind Source Separation for Digital Communications[J]. Electronic Design Engineering, 2009, 17(5): 94-99.

[4]Chen Li. Research on Anti-jamming in Frequency Hopping Communication Systems Based on Blind Source Separation[D]. Chengdu: University of Electronic Science and Technology of China,2014

[5]YAO Jun-liang,YANG Xiao-niu,LI Jian-dong,et al.Performance Analysis of the FastICA Algorithm in Co-channel Communication System[J].Signal Process, 2010,26(5):771-777.

[6]L Wang ,J Karhunen. A unified neural bigradient algorithm for robust PCA and MCA[J].Iat.J.Neural Syst.,1996,7:53-67.

[7]A. Hyvarinen. Fast independent component analysis with noisy data using gaussian moments.In Proc.lnt.Symp.,On Circuits and Systems,1999,V57-V61.

[8]Huang Gao-ming. Research on Radar Signal Sorting Based on Blind Signal Separation[J].Radio Engineering,2004,34(8):30-38. 